\title{
Water migration in wood during imbibition assessed by X-ray imaging
}

\author{
Benoît Martin ${ }^{1,2 *}$, Julien Colin ${ }^{2,3}$, Pin $\mathrm{Lu}^{2}$, Mahamadou Mounkaila ${ }^{2}$, Joel Casalinho ${ }^{3}$, Patrick Perré2,3, and Romain \\ Rémond ${ }^{1}$ \\ ${ }^{1}$ LERMAB, Université de Lorraine, ENSTIB, Epinal, France \\ ${ }^{2}$ Université Paris-Saclay, CentraleSupélec, Laboratoire de Génie des Procédés et Matériaux, SFR Condorcet FR CNRS 3417, \\ Centre Européen de Biotechnologie et de Bioéconomie (CEBB), Pomacle, France \\ ${ }^{3}$ Université Paris-Saclay, CentraleSupélec, Laboratoire de Génie des Procédés et Matériaux, Gif-sur-Yvette, France
}

\begin{abstract}
In the wooden construction, structural elements are particularly exposed to external climatic conditions during the construction phase. To ensure their durability over their expected lifespan, the required drying time have to be known after a rain shower and before closing the timber structures. This question, involving coupled heat and mass transfer, can be investigated through numerical tools. The aim of this work is to provide experimental data through X-ray imaging to validate the prediction potential of computational models simulating transfer in porous media. Two complementary methods were developed to access the water migration in Norway spruce. The first one allowed to quantitatively measure spatial distribution of moisture content over time. It is based on a rigorous treatment of the X-ray beam attenuation. The second method, using a high-resolution 3D reconstruction, highlighted the role of wood rays on liquid migration within the porous network.
\end{abstract}

\section{Introduction}

The number of high-rise wooden buildings has increased significantly in recent years with the democratization of Cross Laminated Timber (CLT) panels [1]. To ensure their correct implementation, moisture content has to remain close to their intended use [2]. Indeed, the structural elements can be exposed to external climatic conditions during the construction phase, which can result in significant variations of moisture content. As a result, dimensional changes occur, causing swelling and internal stresses that can lead to cracks [3]. In addition, engineered wood products may be exposed to leaks from the exterior envelope or water supply system during the life span of the building [4]. These localized increases in moisture content (MC) within envelopes can create favourable conditions for biological degradation when they remain close to the fibre saturation point for several weeks $[5,6]$. To address these issues, numerical tools can be helpful to estimate the necessary drying time or to design composite walls. However, discrepancies between measurements and simulations $[7,8]$. Therefore, a better understanding of the water migration in wood, involving coupled heat and mass transfers, is required for improving numerical models. To do so, the MC distribution in wood has to be assessed accurately over time [9-11].

Oven-dry method (or gravimetric method) is commonly used but give only an average $\mathrm{MC}$ of the entire sample [12]. By cutting the sample in several layers and weighting them, a coarser MC distribution can be obtained, but this procedure is destructive and tedious
[13]. The electrical technique consists of measuring the electrical resistance between moisture pins inserted in a piece of wood. This method is relevant for in situ measurements but gives only a local value of MC. Moreover, the user has to be aware that the measuring range is reliable (from 7 to $30 \%$ ) and related to the electrode position regarding the wood grain orientation $[14,15]$. Nuclear Magnetic Resonance (NMR) is an interesting technique, non-invasive and non-destructive, used in laboratory. It is based on the time relaxation of hydrogen protium $\left({ }^{1} \mathrm{H}\right)$ after being exposed to a magnetic field $[16,17]$. The anatomy structure is not available, but the different stage of water in wood (bound water and free water) can be assessed according to the relaxation time $[18,19]$. To observe the MC distribution qualitatively in a small sample, Resonance Magnetic Imaging (MRI) can be used [20]. However, the time acquisition does not allow to visualize dynamic phenomenon with high resolution. Attenuation of ionizing radiation X-ray or neutron as $\mathrm{RMN}$ is a non-destructive and non-invasive technique. Numbers of works have shown their great potential in observing the liquid water migration in wood and others materials [21-23]. However, the specificities of wood material (hygroscopicity, shrinkage, wettability, etc.) and the protocol to obtain an accurate $\mathrm{MC}$ distribution remain the main challenge for dynamic moisture transfer measurement.

In this work, X-ray imaging was used to assess moisture migration in Norway spruce during imbibition. Two complementary methods have been developed. The

\footnotetext{
${ }^{*}$ Corresponding author: b.martin@univ-lorraine.fr
} 
first method was based on 2D projections to measure the evolution of the MC field over time. For that purpose, a rigorous X-ray beam attenuation treatment was implemented to distinguish water from lignocellulosic material. As the anatomical structure is not accessible in this case, a second experimental method has been developed to visualize liquid water migration within the porous network, using 3D imaging.

\section{Materials and methods}

\subsection{Materials}

Norway spruce (Picea abies), common species in wooden constructions, was studied. The samples were extracted from a board $\left(2000 \times 194 \times 35 \mathrm{~mm}^{3}\right)$ of a 73 years tree harvested in Auvergne (France). Samples from both experiments were extracted in the heartwood. For this work, the imbibition phenomenon was studied in the longitudinal direction. For 2D (imaging, a SECOTOM 50 (Struers) precision cutting machine was used to obtain samples with the following dimensions: $30(\mathrm{~L}) \times 9(\mathrm{R}) \times 3(\mathrm{~T})$ $\mathrm{mm}^{3}$. For 3D imaging, a small cylinder with a diameter of $\emptyset 2.8 \mathrm{~mm}$ and $18 \mathrm{~mm}$ in the longitudinal direction was cut with a CNC machine.

Surfaces parallel to the direction of water uptake were covered with an impermeable sealant (AquaStop v33) to study one-dimensional moisture transfer by limiting mass transfer through the lateral faces of the sample. Before the experiments, the samples were stored in a climatic chamber set at $25^{\circ} \mathrm{C}$ and $50 \%$ relative humidity until a constant mass was reached to ensure a homogeneous initial moisture content.

\subsection{Experimental setup}

Imbibition experiment consisted in putting the bottom surface of a wood sample with liquid water for $24 \mathrm{~h}$. X-ray imaging was performed with the last generation nanotomograph offering a large range of possibilities, from high-resolution imaging to fast imaging (RX Solutions Easy XL Ultra 150-160 with controlled environment). The scanner was equipped with a 16 bits CCD detector, with $2016 \times 1334$ resolution and $18 \mu \mathrm{m}$ pixel size. Environmental conditions during the experiment were monitored using an SHT75 capacitive sensor (Sensirion $\mathrm{AG})$.

\subsubsection{D imaging}

The protocol developed in 2D imaging was based on 2D projections (or radiography) allowing high frequencies acquisition to access the fast liquid migration through the material. In this case, the spatial resolution of the projection is a function of the detector resolution $(9 \mu \mathrm{m})$ and the distances between X-ray source, detector and studied sample as shown in [22,24]. To limit edgeresponse function (ERF) due to the conicity of the incident X-ray beam, the experimental set-up was positioned far from the source and close to the detector (Fig. 1). A $2 \times 2$ binning was used to reach this high acquisition frequency. In this configuration, the magnification equalled to 1.035 , and the spatial resolution of the picture is $17.4 \mu \mathrm{m}$. The sample holder was designed to keep the upper surface entirely free for mass transfers. Particulars attentions were paid to maintain a constant water level in contact with the bottom surface sample.

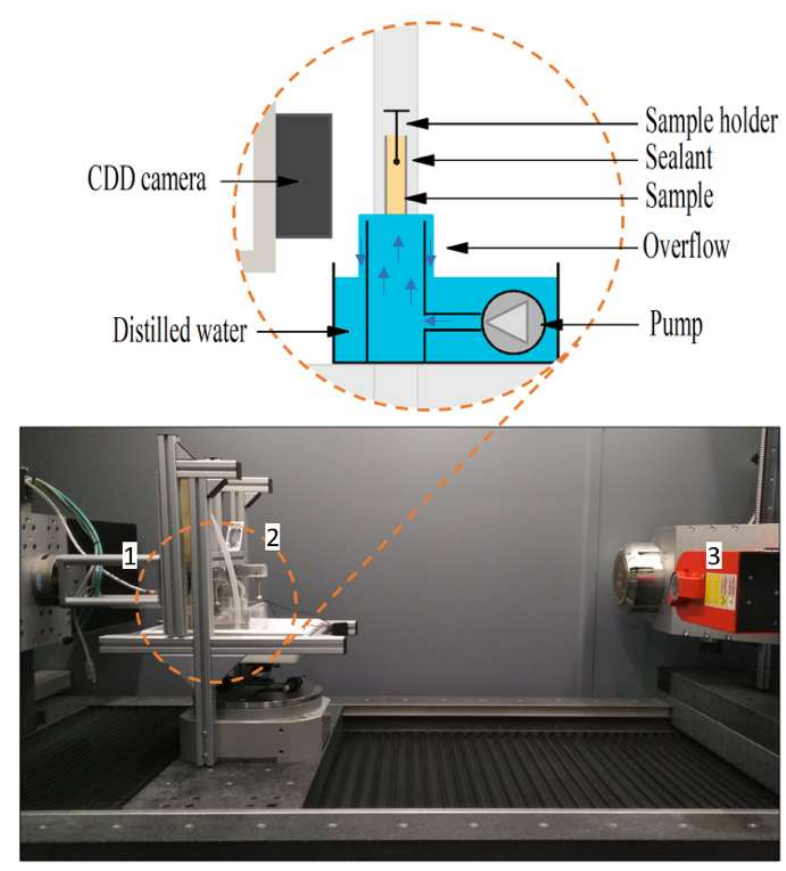

Fig. 1. General view of the experimental setup inside the tomograph: (1) CCD detector, (2) sample holder with water system supply and (3) $\mu \mathrm{X}$-ray source.

The polychromatic X-ray micro-source was set at 90 $\mathrm{kV}$ and $200 \mu \mathrm{A}$. Calibration of the device was required before proceeding to the experiment. This procedure was carried out in two steps. One by recording ten projections without X-ray emission to account for the background environment or background noise (black calibration, $I_{B}$ ). Then, ten more projections, without sample between the detector and the turned-on X-ray source, to obtain a white image representing the spatial distribution of the X-ray source (gain calibration, $I_{G}$ ) [25]. Subsequently, continuous acquisition of the $I$ projections during water absorption was recorded every 10 seconds for the first four hours and after about 24 hours. 24 hours.

\subsubsection{Acquisition for $3 D$ imaging}

In the case of 3D imaging, a similar water supply system has been designed to bring the bottom of a small cylinder into contact with liquid water. The challenge was to make rotation possible during imbibition. The specimen was positioned at the top of an aluminium tube linked to a water container by a flexible tube. The control of the water level in contact with the bottom of the sample was ensured using the system of communicating vessels. (Fig. 2). An $\mathrm{X}$-ray nano-source set at $50 \mathrm{kV}$ and $50 \mu \mathrm{A}$ was used in combination with the same CCD detector. The exposure time was set at 1 second, without any average frame. These parameters made it possible to have a voxel size equal to $3 \mu \mathrm{m}$ with $3 \mathrm{~mm}$ height of the studied volume in a limited time of 30 minutes. 


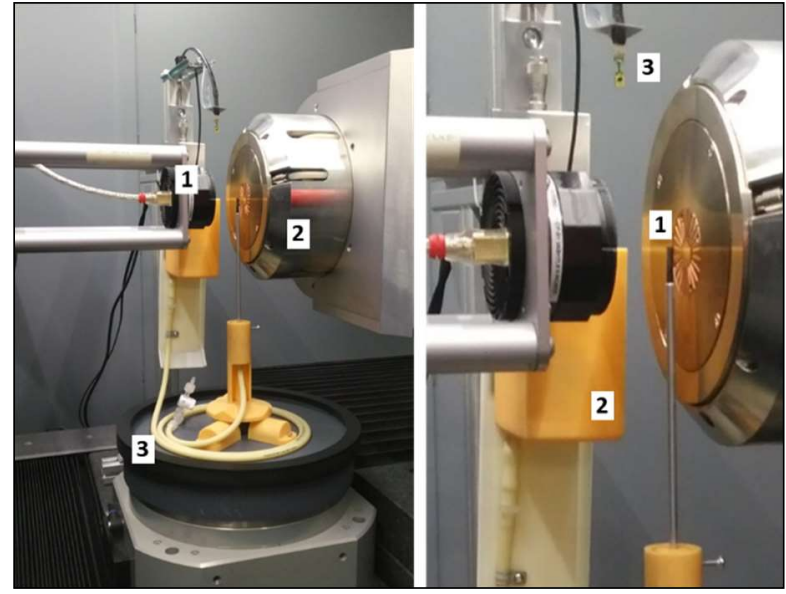

Fig. 2. Experimental setup for 3D imaging with a general view on the left with CCD detector (1), nano X-ray source (2) and flexible tube (3). On the right, a zoom of the sample positioned at the top of aluminium tube (1), a water container (2) and a SHT75 sensor (3).

As for 2D imaging, black and gain calibrations were performed. The locations of two studied areas were chosen at $5 \mathrm{~mm}$ and $15 \mathrm{~mm}$ from the bottom of the sample. A first scan was performed with the sample at the equilibrium moisture content (EMC) with ambient air. Then a series of three scans were performed after contact with water.

\subsection{Post-processing and analysis tools}

\subsection{1 $2 D$ post-processing}

\section{Image processing}

Image processing is a mandatory step before qualitative and quantitative image analysis. A rigorous treatment of the transmission imaging of a polychromatic X-ray beam was implemented including:

- $\quad$ Denoising of the raw projections $I, I_{B}$ and $I_{G}$ by calculating the median value over ten projections for each pixel $(\mathrm{i}, \mathrm{j})$ to obtain $I^{\prime}, I_{B}^{\prime}$, and $I_{G}^{\prime}$

- $\quad$ Subtraction of dark noise $I_{B}^{\prime}$ to the projections $I^{\prime}$ and $I_{G}^{\prime}$

- Correction of the non-uniformity X-ray beam by applying a factor $f$ to set the pixel $(\mathrm{i}, \mathrm{j})$ equal to the mean of grey level of the projection $I_{G}^{\prime}$

The interest in image processing is illustrated in Fig. 3 with the grey level distribution of the projection before (Fig. 3-A) and after application treatment (Fig. 3B). In projection $I^{\prime \prime}$ (Fig. 3-B), the first peak (around 2000) corresponds to the sample holder, the peak around 8000 to the gain (ambient air) and, in between, the wood sample. The greyscale distribution of the wood consists of a peak corresponding to the earlywood followed by a shoulder corresponding to the latewood (darker grey than earlywood) due to a gradual transition during one annual growth.
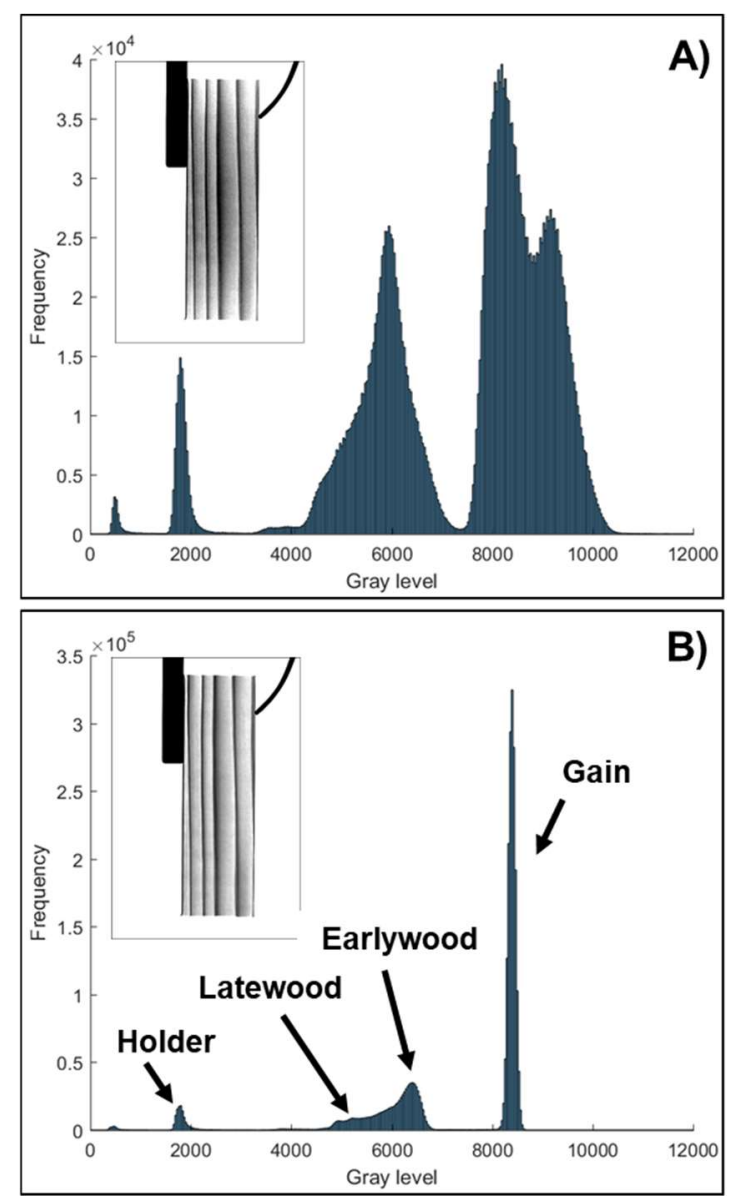

Fig. 3. Distribution of grey level before (A) and after (B) image processing

\section{Moisture content measurement}

The measurement of MC is carried out in two stages: (i) measuring the $\mathrm{X}$-ray attenuation of the sample for a given and homogeneous MC, (ii) then measuring the variation of X-ray attenuation that one associate with a variation in areal density in order to determine the evolution of MC field. Attenuation is defined as the ratio of the intensity of the transmitted X-rays through the sample $I_{\text {wood }}^{\prime \prime}$ over the incident X-rays intensity $I_{0}^{\prime \prime}$ (Fig. 5).

$$
A_{(S)}=\frac{I_{\text {wood }}^{\prime \prime}}{I_{0}^{\prime \prime}}
$$

The attenuation $A_{(S)}$ was measured directly on each projection $I^{\prime \prime}$ during imbibition experiment (Fig. 5). The selection of regions of interest (ROI) with a height of $1.4 \mathrm{~mm}$, provided access to the distribution of moisture content along the length of the sample (Fig. 5).

It is, therefore, necessary to know the relationship between the X-ray attenuation (1) and the areal density of wet wood. The X-ray source being polychromatic with an energy-integrating X-ray detector, it is necessary to go through a calibration step of the device to determine the relationship between the X-ray attenuation and the areal density of wet wood [26].

This primary calibration step (i) was performed with parallelepipedal samples of Sorbus torminalis. This specie was chosen because it is a diffuse pore species 
giving it a homogeneous anatomical structure along the annuals growth rings. This characteristic permitted to have samples with the same density whatever their dimensions [27]. For calibration, each of these samples was scanned under two different states: air-dried (MC $\simeq 8 \%$, d.b.) and fully saturated in water (MC $\simeq 125 \%$, d.b.). The MC was measured using a gravimetric method and controlled before and after each acquisition. Their X-ray attenuation is depicted in Fig. 4 according to their equivalent areal density $\rho_{e q}^{*}(2)$. The equivalent areal density includes water and wood material. Indeed, the wet material can be considered as a two-phase component with lignocellulosic material and water having their own density and attenuation. At high energies (from $90 \mathrm{keV}$ ) the water/wood ratio attenuation can be considered constant, which makes it possible to introduce a factor $\alpha$ to link the lignocellulosic material attenuation to water attenuation (11). The areal density of the sealant $\rho_{\text {sealant }}^{*}$, assuming constant, was calculated by measuring the X-ray attenuation of the sample before and after application of the coating, at the same moisture content.

$$
\rho_{e q}^{*}=\rho_{0}^{*} \cdot(x+\alpha)+\rho_{\text {sealant }}^{*}
$$

with $\rho_{e q}^{*}$ the equivalent areal density $\left(\mathrm{kg} / \mathrm{m}^{2}\right), \rho_{0}^{*}$ the areal density of wood $\left(\mathrm{kg} / \mathrm{m}^{2}\right), x$ the moisture content (-), $\alpha$ the factor to link the lignocellulosic material and water attenuation $(-), \rho_{\text {sealant }}^{*}$ the areal density of the sealant $\left(\mathrm{kg} / \mathrm{m}^{2}\right)$

A non-linear model proposed in [27] was used to link the logarithm attenuation (3) with the equivalent areal density $\rho_{e q}^{*}(2)$. The values of parameters $L_{0}, L_{1}, \alpha$ and $\lambda$ were identified by minimizing the gap between the numerical and the experimental data (least-squares method).

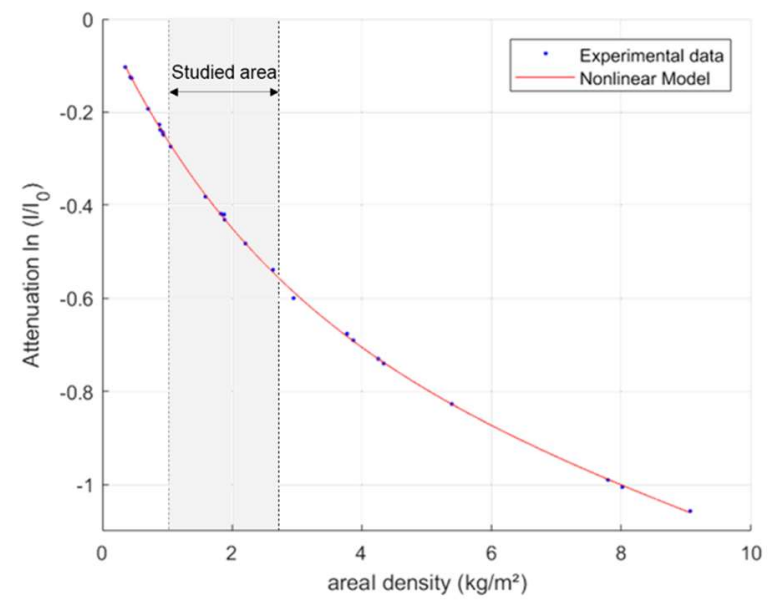

Fig. 4. Experimental data from Sorbus samples of different thicknesses and moisture content with the non-linear fitting curve. Studied are represented in grey.

$$
\ln \left(A_{(S)}\right)=L_{0} \cdot \rho_{e q}^{*}+L_{1} \cdot\left(1-\exp ^{\left.\left(-\rho_{e q}^{*}\right) / \lambda\right)}\right)
$$

where $L_{0}=-0,04713 ; L_{1}=-0,65172 ; \lambda=$ 2,54123 and $\alpha=0,71229$
The three first values $\left(L_{0}, L_{1}\right.$ and $\left.\lambda\right)$ depend on source/detector settings and $\alpha$ is assumed to constant whatever the species. Indeed, the elementary composition of the lignocellulosic material is relatively stable from one species to another.

After calibration, this model can be used for determining the spatial distribution of moisture content over time from the series of images obtained during imbibition (ii). In the expression (3), the only unknown variable was the moisture content $\mathrm{x}$, which can be identified using a nonlinear solver.

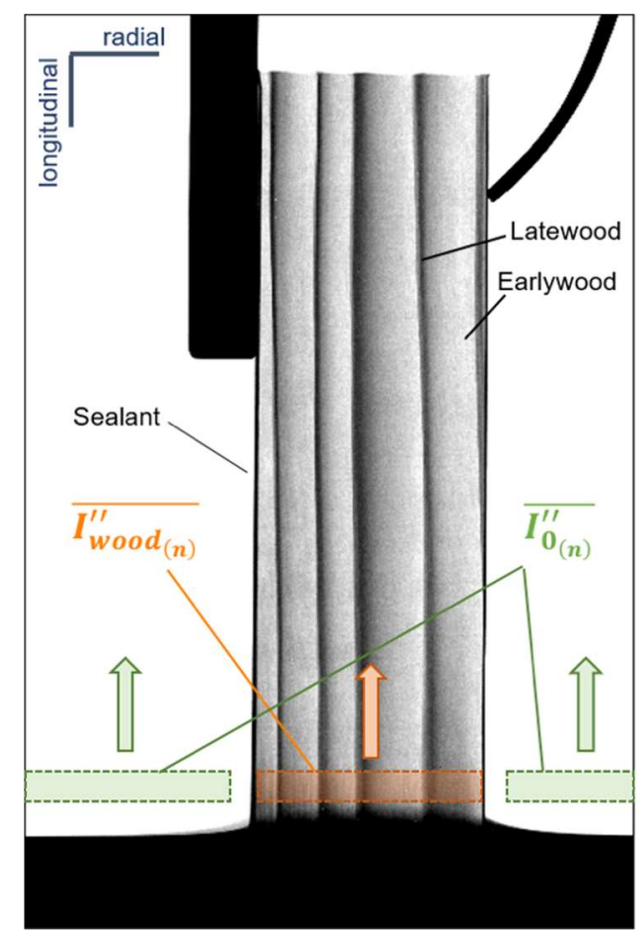

Fig. 5. Selected regions of interest for the calculation of attenuation $A_{(S)}$

\subsubsection{D reconstruction and analysis methods}

The 3D reconstructions were carried out using $\mathrm{RX}$ Solution X-act. The stack of slices obtained along the zaxis was processed using ImageJ. First, a circular ROI, taken into account the swelling, was automatically defined to exclude the coating on the periphery. Second, a local threshold was applied to distinguish air, on the one hand, from wood or water, on the other hand. The attenuation of X-rays from water and wood is close, which does not allow to differentiate them by thresholding. Third, isolated pixels corresponding to the acquisition noise were removed. Finally, the slices thus obtained after treatment allowed to visualize the filled lumens (black) of those which are empty (white).

\section{Results and discussions}

\subsection{D imaging results}

Fig. 6 shows the initial wood state and the migration of water for three selected times during the test. The 
migration front was visible from the first projection I", i.e. after 50 seconds (Fig. 6-b). Then, its progression in the sample is non-homogeneous with a different spatial distribution between the annual rings, as observed in other works [21,28]. The moisture absorption kinetics measured for five ROI (along the z-axis) are plotted in Fig. 7 The dashed line represents the average MC of all ROI. The initial MC was $11,7 \%$ (assumed uniform). No significant change of MC was measured and qualitatively observed in the ROI $\mathrm{n}^{\circ} 15$ and 20 located at 23.70 and $29.12 \mathrm{~mm}$ from the bottom. On the other hand, the ROI $n^{\circ} 1$ located at $3.35 \mathrm{~mm}$ to the bottom reaches the fibre saturation point $(\mathrm{MC}=31,5 \%)$ after 50 seconds and $110.7 \%$ at the end of the experiment. The moisture absorption kinetics for this ROI is composed of two phases mainly. A high rate of water migration characterized the first imbibition phase during the early stages, followed by a lower increase in moisture content. In ROI No. 5 at $8.95 \mathrm{~mm}$, the moisture content increased but to a lesser extent than the ROI $\mathrm{n}^{\circ} 1$. This kinetics can be explained by both $(i)$ a heterogonous free water uptake where only the left side of the sample where reached by the water and (ii) the low diffusive flux due to the distance from ROI No. 5 to the wet surface (Fig. 6).
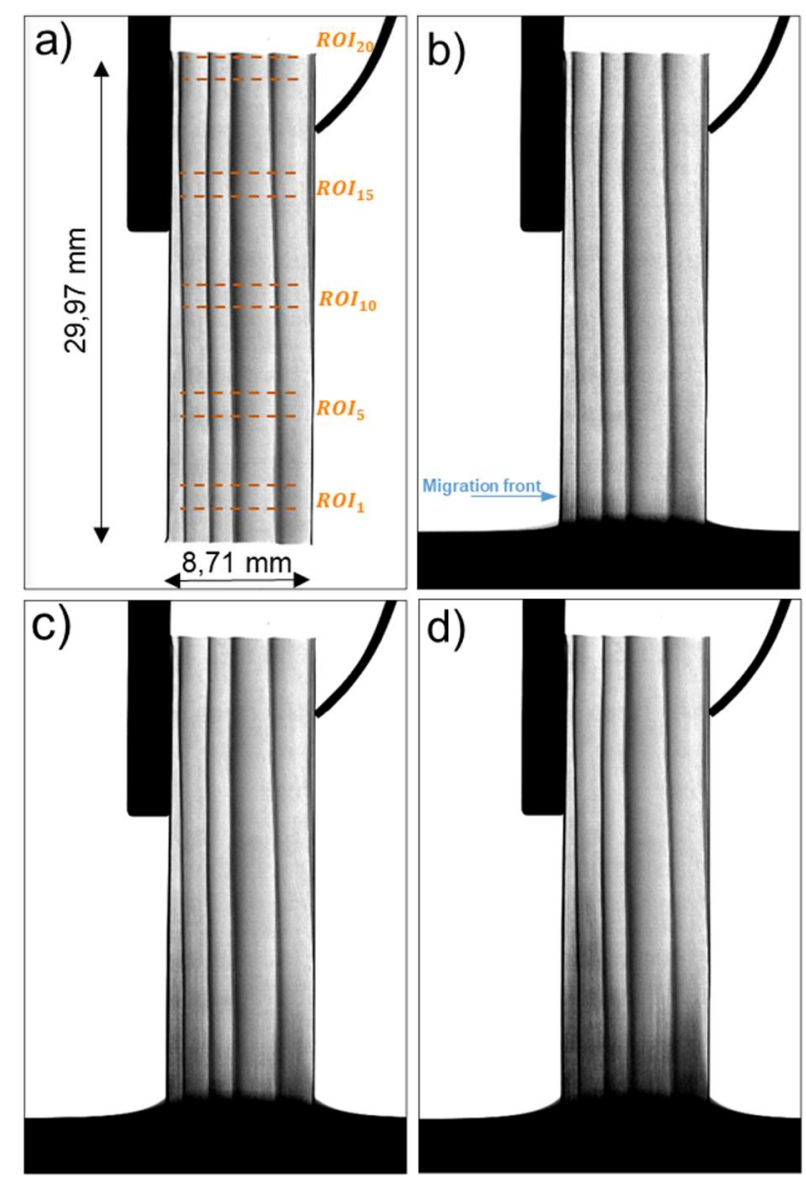

Fig. 6. Water uptake in spruce along longitudinal direction at $\mathrm{t}_{0}(\mathrm{a}), \mathrm{t}_{50 \mathrm{~s}}(\mathrm{~b}), \mathrm{t}_{4 \mathrm{~h} 01}(\mathrm{c})$ and $\mathrm{t}_{23 \mathrm{~h} 18}(\mathrm{~d})$

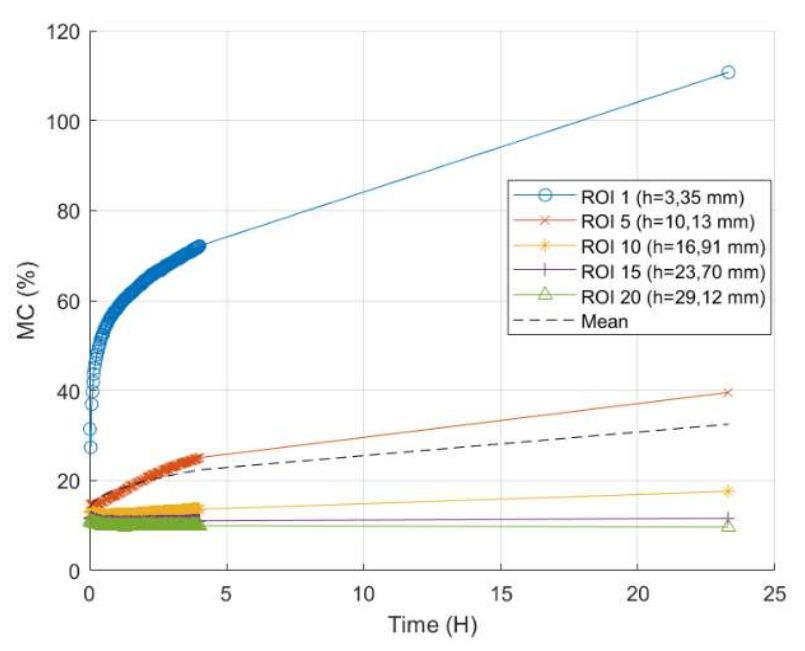

Fig. 7. Moisture content kinetic measured on ROI located at $3.35,10.13,16.91,23.70$ and $29.12 \mathrm{~mm}$ from bottom in contact with liquid water

\subsection{D imaging results}

Fig. 8 shows cross-sections positioned at the height of 5 and $15 \mathrm{~mm}$ from the bottom surface in contact with the liquid water. The position of the $3 \mathrm{D}$ reconstruction at 5 $\mathrm{mm}$ was chosen to be close to the surface in contact with water but at least above the first tracheid layer, which is about $3 \mathrm{~mm}$ long. The cross-sections at several times $\mathrm{t}_{0}$, $\mathrm{t}_{1 \mathrm{~h} 18}, \mathrm{t}_{3 \mathrm{~h}} 45$ and $\mathrm{t}_{20 \mathrm{~h}} 36$ show a complex migration of liquid water in the porous network. No preferential migration between the earlywood and the latewood, as mentioned in other works [23,29] were observed. The tortuous migration of water through the porous network can be explained by the random embolism of the punctuations that creates a partially connected porosity. To better understand this migration, and investigate the dissimilar behaviour regarding previous studies [20,23,28], sections were made according to the $L / R$ plane of the $3 D$ reconstruction, without any preliminary image processing (Fig. 9). The location of the AA sections can be seen in Fig. 8. Within tracheid lumens, a number of gas inclusions between two liquid water volumes were observed. This investigation shows that wood rays play an important role in the liquid migration of water via the cross-field pits. It is also interesting to observe a water/wood contact angle within the lumens which confirm cell wall wettability.

\section{Conclusion and perspectives}

This work aimed to use the potential of X-ray imaging to assess the movement of liquid water in Norway spruce during imbibition in the longitudinal direction. Two complementary methods were developed. The first one based on 2D projections giving access to the dynamics of moisture migration in the early stages. A rigorous X-ray attenuation treatment protocol was implemented and validated. 


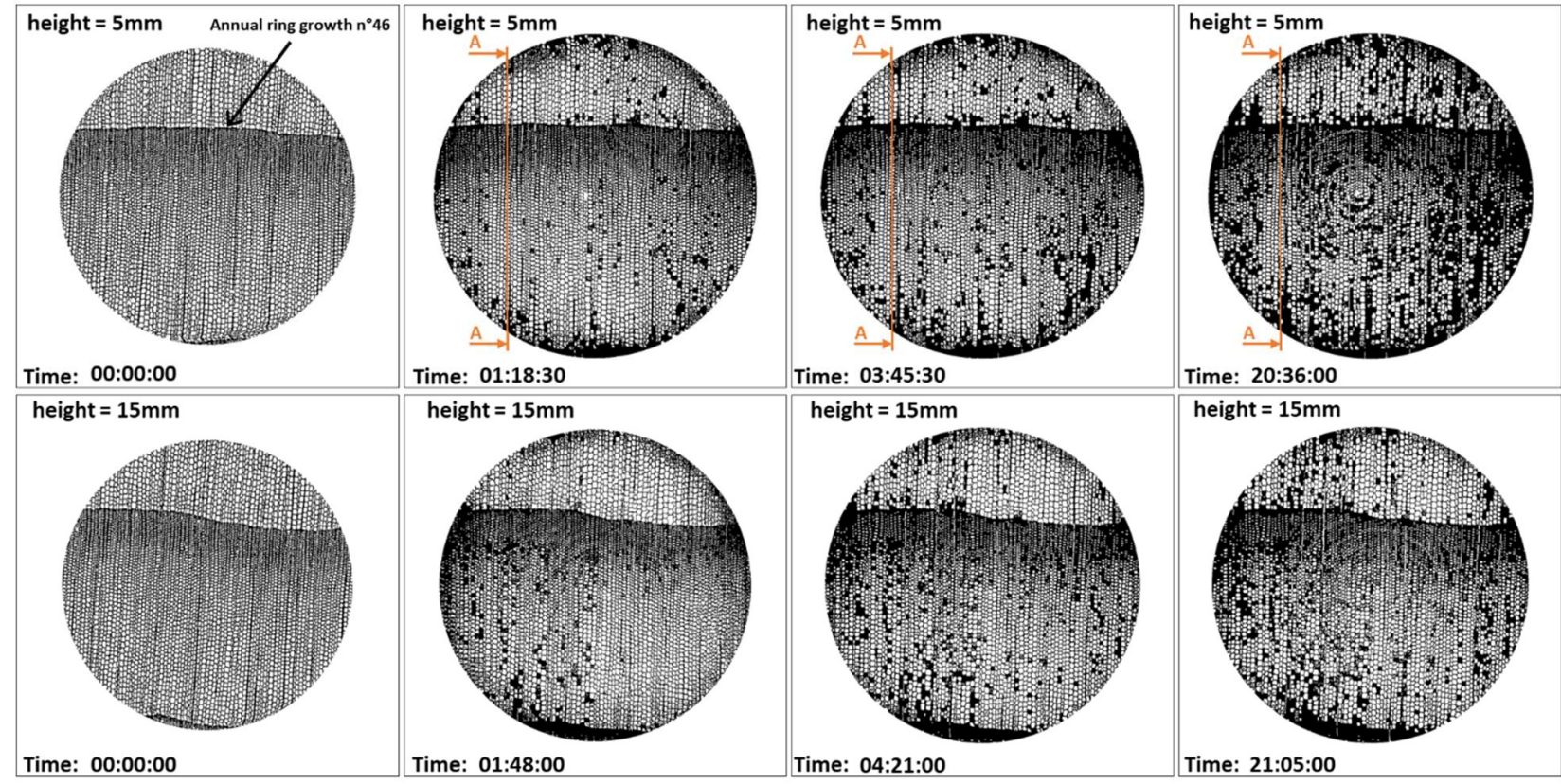

Fig. 8. Cross section located at 5 (top) and $15 \mathrm{~mm}$ (bottom) from liquid contact water during imbibition

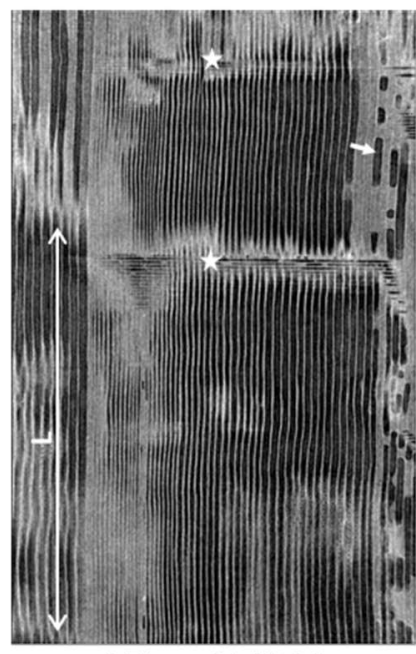

a) Time: 01:18:30

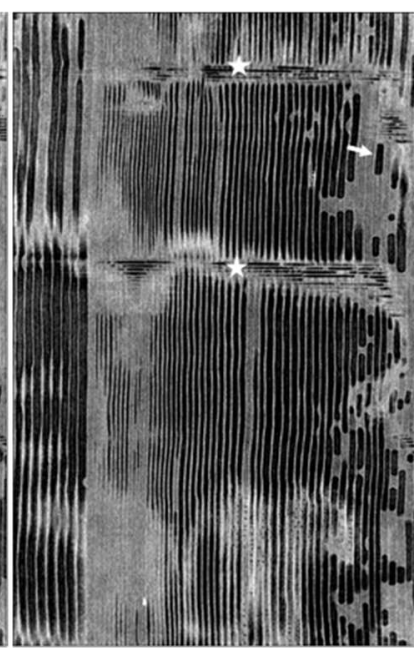

b) Time: 03:45:30
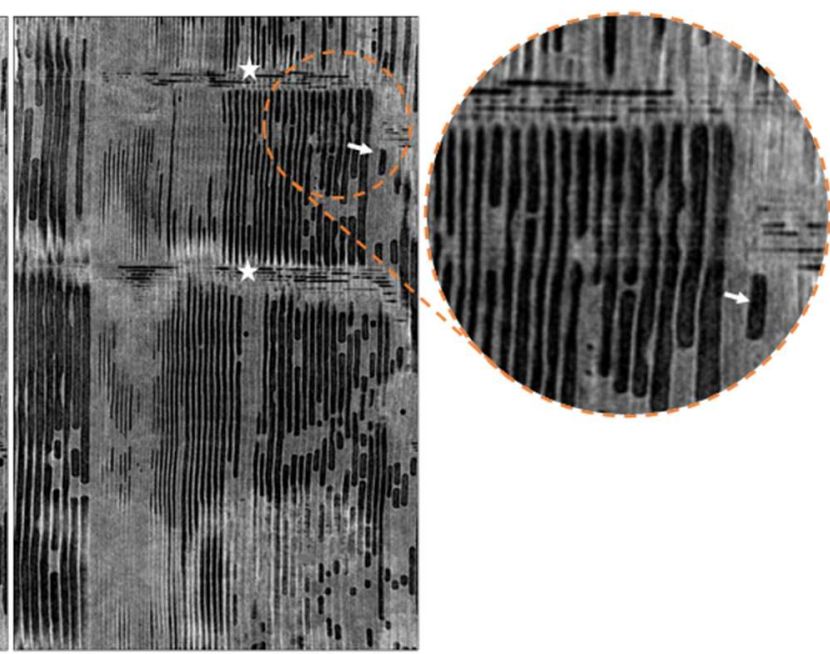

Fig. 9. Sections AA located at $5 \mathrm{~mm}$ from water contact at different times. Stars represent the location of wood rays, L the length of one tracheid and arrows the evolution of liquid water in a local area with gas pocket

The second method uses a high-resolution 3D reconstruction (voxel size $=3 \mu \mathrm{m}$ ) from a series of $2 \mathrm{D}$ projections. After image processing, the cross-section of the stack indicates a complex migration in the pore network

No preferential pathway was observed between earlywood and latewood on the test carried out, which seems to be in contradiction with previous work. The location of embolism pits may explain the spatial distribution of water-filled wood which also migrate through the wood rays as visible on the cross-section. Furthers investigations have to be performed to study the wood surface quality in contact with the liquid water, which may have been damaged during machine processing, that may thus play a role in the first millimetres of liquid water migration.
The tools and methods developed in this work are of great help to follow the evolution of humidity through wood cells. Future work will focus on latewood and earlywood migration using both methods. Imbibition tests, according to the radial direction, will also be studied in spruce and other wood species with higher liquid permeability.

The authors would like to thank Région Grand Est, Département de la Marne, Grand Reims and the European Union for supporting the Centre Européen de Biotechnologie et de Bioéconomie (CEBB 51110 Pomacle, France), where this study was carried out. In particular, the authors would like to thank Département de la Marne for its financial support.

For four years (from 01/05/2016 to $31 / 07 / 2020$ ) and with a total budget of $965,000 €, 3 \mathrm{D}$-BioMat is co-financed by the Grand Reims ( $31 \%$ ) and the European Union by $48.7 \%$ (i.e. 
$50 \%$ of eligible expenditure). Europe is committed to the Grand Est with the European Regional Development Fund.

ADEME is a partner of the project and co-finances a thesis scholarship within the framework of the theme "sustainable cities and territories", contribution to the development of highperformance buildings integrating biosourced materials.

The project benefits from government aid managed by the French National Research Agency under the Investissements d'avenir programme bearing the reference no. ANR-11LABX-0002-01 (Laboratoire d'Excellence ARBRE).

\section{References}

1. R. Brandner, G. Flatscher, A. Ringhofer, G. Schickhofer, and A. Thiel, Eur. J. Wood Wood Prod. 74, 331 (2016)

2. R. Lepage, Moisture Response of Wall Assemblies of Cross-Laminated Timber Construction in Cold Canadian Climates, University of Waterloo, 2012

3. E. L. Schmidt, M. Riggio, A. R. Barbosa, and I. Mugabo, Build. Environ. 148, 609 (2019)

4. A. Tietze, S. Ott, S. Boulet, K. Gradeci, N. Labonnote, S. Grynning, J. Noreen, and Pousette Anna, Tall Timber Facades - Identification of Coss-Effective and Resilient Envelopes for Wood Constructions (TallFacades) (2017)

5. H. Viitanen, Mater. Struct. 27, 483 (1994)

6. P. Johansson, G. Bok, and A. Ekstrand-Tobin, Build. Environ. 65, 178 (2013)

7. K. Krabbenhoft and L. Damkilde, Wood Sci. Technol. 38, 641 (2004)

8. J. Johansson and G. Kifetew, Eur. J. Wood Wood Prod. 68, 77 (2010)

9. P. Niemz and D. Mannes, J. Cult. Herit. 13, S26 (2012)

10. P. Perré, Dry. Technol. 29, 1529 (2011)

11. M. C. Phillipson, P. H. Baker, M. Davies, Z. Ye, A. McNaughtan, G. H. Galbraith, and R. C. McLean, Build. Serv. Eng. Res. Technol. 28, 303 (2007)

12. NF EN 13183-1, Moisture Content of a Piece of Sawn Timber - Part 1: Determination by Oven Dry Method (2002)

13. M. de Meijer and H. Militz, Holz Als RohWerkst. 58, 354 (2000)

14. NF EN 13183-2, Moisture Content of a Piece of Sawn Timber - Part 2: Estimation by Electrical Resistance Method (2002)

15. M. Tiitta and H. Olkkonen, Rev. Sci. Instrum. 73, 3093 (2002)

16. S. Hameury and M. Sterley, Wood Mater. Sci. Eng. 1, 116 (2006)
17. L. Passarini, C. Malveau, and R. E. Hernández, Wood Sci. Technol. 49, 1251 (2015)

18. Ö. Gezici-Koç, S. J. F. Erich, H. P. Huinink, L. G. J. van der Ven, and O. C. G. Adan, Cellulose 24, 535 (2017)

19. G. Almeida, S. Gagné, and R. E. Hernández, Wood Sci. Technol. 41, 293 (2007)

20. M. Zhou, S. Caré, D. Courtier-Murias, P. Faure, S. Rodts, and P. Coussot, Wood Sci. Technol. 52, 929 (2018)

21. D. Derome, S. Roels, and J. Carmeliet, 9 (2005)

22. S. Roels and J. Carmeliet, Int. J. Heat Mass Transf. 49, 4762 (2006)

23. M. Sedighi-Gilani, M. Griffa, D. Mannes, E. Lehmann, J. Carmeliet, and D. Derome, Int. J. Heat Mass Transf. 55, 6211 (2012)

24. K. Watanabe, Y. Saito, S. Avramidis, and S. Shida, Dry. Technol. 26, 590 (2008)

25. E. Badel and P. Perré, Ann. For. Sci. 59, 767 (2002)

26. B. J. Pease, G. A. Scheffler, and H. Janssen, Constr. Build. Mater. 36, 419 (2012)

27. A. Bouali, R. Rémond, G. Almeida, and P. Perré, in (Xiamen, China, 2012)

28. K. Sandberg and J.-G. Salin, Wood Sci. Technol. 46, 207 (2012)

29. G. Almeida, S. Leclerc, and P. Perre, Int. J. Multiph. Flow 34, 312 (2008) 